\title{
Interchange instability of the plasma disk in Jupiter's middle magnetosphere and its relation to the radial plasma density distribution
}

\author{
P. A. Bespalov ${ }^{1}$, S. S. Davydenko ${ }^{1}$, S. W. H. Cowley ${ }^{2}$, and J. D. Nichols ${ }^{2}$ \\ ${ }^{1}$ Institute of Applied Physics, Russian Academy of Sciences, 46 Ulyanov St, 603950 Nizhny Novgorod, Russia \\ ${ }^{2}$ Department of Physics \& Astronomy, University of Leicester, Leicester LE1 7RH, UK
}

Received: 24 November 2005 - Revised: 19 April 2006 - Accepted: 23 June 2006 - Published: 9 August 2006

\begin{abstract}
We analyse the interchange or flute instability of the equatorial plasma disk in Jupiter's middle magnetosphere. Particular attention is paid to wave coupling between the dense plasma in the equatorial disk and the more rarefied plasma at higher latitudes, and between the latter plasma and the conducting ionosphere at the feet of the field lines. It is assumed that the flute perturbations are of small spatial scale in the azimuthal direction, such that a local Cartesian approximation may be employed, in which the effect of the centrifugal acceleration associated with plasma rotation is represented by an "external" force in the "radial" direction, perpendicular to the plasma flow. For such small-scale perturbations the ionosphere can also be treated as a perfect electrical conductor, and the condition is determined under which this approximation holds. We then examine the condition under which flute perturbations are at the threshold of instability, and use this to determine the corresponding limiting radial density gradient within the plasma disk. We find that when the density of the high-latitude plasma is sufficiently low compared with that of the disk, such that coupling to the ionosphere is not important, the limiting radial density profile within the disk follows that of the equatorial magnetic field strength as expected. However, as the density of the high-latitude plasma increases toward that of the equatorial disk, the limiting density profile in the disk falls increasingly steeply compared with that of the magnetic field, due to the increased stabilising effect of the ionospheric interaction. An initial examination of Galileo plasma density and magnetic field profiles, specifically for orbit G08, indicates that the latter effect is indeed operative inside radial distances of $\sim 20 R_{J}$. At larger distances, however, additional density smoothing effects appear to be important.
\end{abstract}

Keywords. Magnetospheric physics (Magnetospheric configuration and dynamics; MHD waves and instabilities; Planetary magnetospheres)

Correspondence to: P. A. Bespalov

(pbespalov@mail.ru)

\section{Introduction}

A dominant feature of Jupiter's middle magnetosphere is the equatorial disk of plasma produced from the atmosphere of the moon Io, which orbits deep within the equatorial magnetosphere at a radial distance of $\sim 6 R_{J}$, and produces a heavy ion (sulphur and oxygen) plasma whose overall source strength is $\sim 1000 \mathrm{~kg} \mathrm{~s}^{-1}$ (e.g. Hill et al., 1983; Vasyliunas, 1983; Khurana and Kivelson, 1993; Bagenal, 1997; Delamere and Bagenal, 2003). Here $R_{J}$ is Jupiter's radius, equal to $\sim 71400 \mathrm{~km}$. The Io source initially results in the formation of a dense plasma torus in the vicinity of the moon's orbit (Bagenal and Sullivan, 1981; Belcher, 1983; Bagenal, 1994), which rotates with the field in the equatorial plane with an angular velocity which is just a few percent smaller than that of the planet, due to the plasma particle pick-up that occurs within it (Pontius and Hill, 1982; Brown, 1994). However, the torus distribution is unstable to the excitation of flux tube interchange motions, which cause the cool dense plasma to move outward under the action of the centrifugal force, which is stronger than the inward gravitational force at radial distances beyond $\sim 2.2 R_{J}$ (Siscoe and Summers, 1981; Siscoe et al., 1981; Pontius and Hill, 1989; Southwood and Kivelson, 1989; Kivelson et al., 1997; Thorne et al., 1997). As it does so, plasma corotation with the planet is maintained partially by angular momentum transfer from the atmosphere and ionosphere, resulting in the formation of a large-scale magnetosphereionosphere coupling current system that is associated with Jupiter's main auroral oval (e.g. Hill, 1979, 2001; Pontius, 1997; Cowley and Bunce, 2001; Nichols and Cowley, 2004). The iogenic plasma is believed eventually to be lost by down-tail outflow via the dusk flank, in a process that involves closed field line stretching, pinch-off, and plasmoid formation (e.g. Vasyliunas, 1983; Woch et al., 2002; Kronberg et al., 2005). 


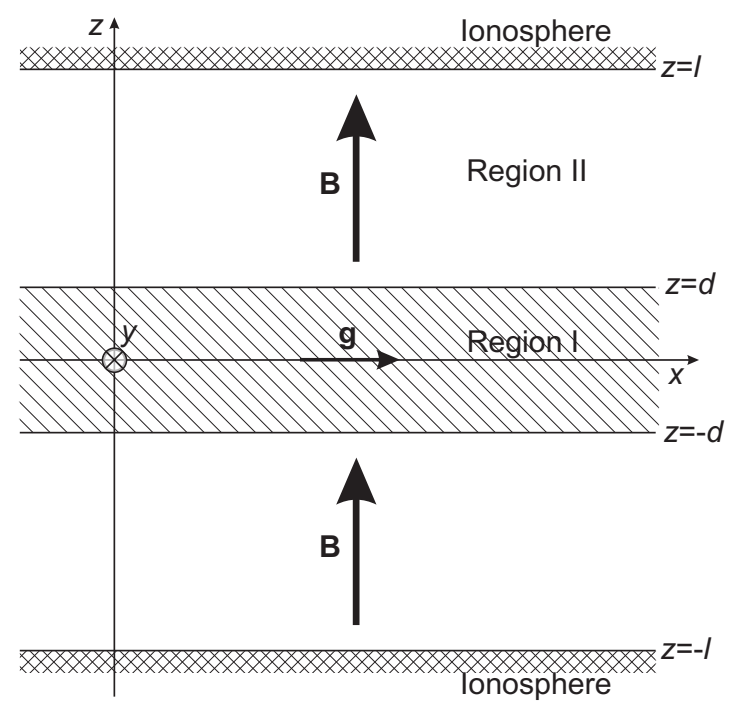

Fig. 1. Sketch of the local Cartesian system analysed in this paper. A slab of high-density iogenic plasma (shown hatched), termed "region I", lies in the $x-y$ plane in the region given by $|z| \leq d$, thus centred on the equatorial plane at $z=0$. Outside the slab lies a more tenuous plasma on either side, termed "region II", which is bounded by the conducting planetary ionosphere at $z= \pm l$. The field lines indicated by the $\boldsymbol{B}$ vector are straight, and pass orthogonally through the equatorial plasma slab between the ionospheres as shown. The unperturbed field and plasma parameters (such as the field strength, field line length, and plasma density) vary only in the $x$ direction, thus representing radial distance from the centre of the planet. The unperturbed plasma convective velocity is then in the $y$ direction (into the plane of the diagram as shown), representing partial plasma corotation with respect to the planetary angular velocity. The acceleration of the disk plasma due to the centrifugal effect of plasma rotation and to field line curvature is then represented by an "external" force $\boldsymbol{g}$ in the $x$ direction, as also shown.

The outward-flowing mass-loaded flux tubes are replaced in the interchange process by "unloaded" flux tubes from the outer regions, which contain a tenuous plasma which is compressed and heated during its inward transport. The co-existing plasma populations to which this process gives rise in the jovian middle magnetosphere thus consist of two components. The first is cool dense iogenic plasma, with energies typically below, say, $\sim 10 \mathrm{keV}$, which diffuses outwards, mainly confined to near the equatorial plane by centrifugal action (e.g. Belcher, 1983; Bagenal, 1994; Frank and Paterson, 2001, 2004). The density of this cool equatorial plasma is observed to fall, at radial distances between $\sim 10$ and $\sim 50 R_{J}$, approximately as a power law of the distance, with an exponent of $\sim 4-5$ (e.g. Gurnett et al., 1981; Divine and Garrett, 1983). The second is a hot tenuous plasma more broadly distributed along the field lines, with energies above $\sim 10 \mathrm{keV}$, reaching to $100 \mathrm{~s} \mathrm{keV}$ in the torus region, which diffuses inward (e.g. Krimigis and Roelof, 1983; Mauk et al., 1996; Woch et al., 2004). The centrifugal action of the cool component, which contains most of the plasma mass, combined with the pressure gradient of the hot component, which contains most of the plasma thermal energy, then act to stretch the magnetic field lines outward away from the planet, associated with an azimuthal current, which is a characteristic feature of the middle magnetosphere region (Acuña et al., 1983; Mauk et al., 1985; Caudal, 1986; Bunce and Cowley, 2001; Khurana, 2001).

Although this general picture of the physics of Jupiter's middle magnetosphere has been current for a significant period, a detailed understanding of the nature of the associated transport processes, and their relation to the plasma properties, has proven elusive. Initial discussions were based on interchange motions of whole flux tubes, using either assumed or observed distributions of field and plasma (e.g. Gold, 1959; Melrose, 1967; Hill, 1976; Goertz, 1980; Hill et al., 1981; Summers and Siscoe, 1985; Pontius et al., 1986; Southwood and Kivelson, 1987; Pontius and Hill, 1989; Huang and Hill, 1991). The results of related computer simulations have also been presented (Yang et al., 1994; Pontius et al., 1998). More recently, the effect of magnetic perturbations on the interchange or "flute" instability have been discussed by Liu (1998), while Ferrière and André (2003) have discussed a mixed fluid-kinetic approach to low-frequency instabilities.

In this paper we consider the flute instability in a plasma model in which most of the plasma mass is confined near the equatorial plane, as assumed in many of the works cited above for reasons already discussed, but where tenuous plasma is also present outside of the equatorial disk, and account is taken of the communication with the conducting ionosphere at the feet of the field lines. The results of linear stability analysis are used to estimate the equilibrium radial profile of the equatorial plasma, assuming that the growth rate of the most unstable mode is just zero on each field line (e.g. Bespalov and Zheleznyakov, 1990). This estimate provides the steepest radial profile of the plasma density that is stable to the flute modes. This threshold profile can then be further smoothed by slower instabilities, though this aspect is not considered here.

\section{Background plasma model and basic equations}

For mathematical convenience we consider electrostatic perturbations in the plasma system with the simplified Cartesian geometry shown in Fig. 1. The cool dense iogenic equatorial plasma (region I) lies in a slab in the $x-y$ plane of thickness $2 d$, centred on $z=0$. For simplicity of calculation the plasma in this region is taken to consist of one singly-charged ion species, oxygen in numerical estimates, and electrons, and we also ignore the plasma pressure as discussed further below. The magnetic field lines are taken to be straight in the $z$ direction, thus passing normally through the plasma slab, and are bounded by the ionosphere at $z= \pm l$, which has a 
finite height-integrated Pedersen conductivity $\Sigma_{P}$. The field strength $B$ and the unperturbed plasma density $n_{I}^{(0)}$ within the slab vary only in the $x$ direction, which thus represents radial distance from the planet. The unperturbed density of the more rarefied plasma outside the equatorial slab (region II) also varies only with $x$, and is taken to be a fixed fraction of the slab density, so that

$n_{I I}^{(0)}(x)=\tau n_{I}^{(0)}(x)$,

where $\tau$ is a constant less than unity. The unperturbed rotational motion of the plasma is then represented as a velocity in the $y$ direction that depends only on $x$. Here we take this velocity to correspond to essentially rigid corotation of the plasma with the planet out to a distance of $15 R_{J}$, at angular velocity $\Omega_{J}$ (equal to $1.76 \times 10^{-4} \mathrm{rad} \mathrm{s}^{-1}$ ), after which the velocity remains constant at the value $V=15 \Omega_{J} R_{J} \approx 190 \mathrm{~km} \mathrm{~s}^{-1}$ such that the effective angular velocity falls inversely with the distance. This behaviour is based on the velocity measurements obtained from Voyager plasma data by Belcher (1983) and Sands and McNutt (1988). The unperturbed convection velocity is hence given by

$V_{y}=\left\{\begin{array}{ll}\Omega_{J} x & x \leq 15 R_{J} \\ V=15 \Omega_{J} R_{J} & x \geq 15 R_{J}\end{array}\right.$.

The centrifugal acceleration associated with this motion in the real rotational system is then represented by an "external" force per unit mass in the $x$ direction given by

$g_{x}^{c f}=\frac{V_{y}^{2}}{x}$,

which is thus the same for ions and electrons, and for $x \geq 15 R_{J}$ (the main region of interest here) is

$g_{x}^{c f}=\frac{\left(15 \Omega_{J} R_{J}\right)^{2}}{x} \approx 500 \frac{R_{J}}{x} \mathrm{~m} \mathrm{~s}^{-2}$.

For simplicity, this force is ignored for the more tenuous plasma lying outside the slab, an approximation that is appropriate for two reasons. The first is that in the real middle magnetosphere current sheet field geometry the centrifugal force per unit mass transverse to the magnetic field will actually fall significantly with distance from the current sheet along a given field line (in the $z$ direction in our slab model). This is due both to the change in the magnetic field direction relative to the radial vector from the rotation axis, and to the reduction in plasma rotation speed at fixed angular velocity at smaller radial distances from the planet. For simplicity we have thus effectively employed a zero approximation immediately outside of the current sheet. The second is that in the jovian middle magnetosphere, typically only a few percent of the total mass of plasma on a given field line lies outside of the current sheet slab. Consequently, the development of the instability will be dominated by the centrifugal effect of the latter plasma population, as included in our calculation, and the contribution of the plasma mass at higher latitudes will be small.

We also note that despite the fact that the field lines in our simplified model are straight, the effect of the actual curvature of the field within the current sheet can also be incorporated in the model by the inclusion of a second "external" force in the $x$ direction on the plasma in the equatorial slab. Assuming that particle guiding-centre motion is valid within the current sheet, the force per unit mass for particle species $\alpha$ is

$g_{\alpha x}^{\text {cur }}=\frac{v_{T \alpha}^{2}}{R_{c}} \approx \frac{T_{\alpha}}{m_{\alpha} R_{c}}$,

where $R_{c}$ is the radius of curvature of the field lines, and $m_{\alpha}$ is the mass of species $\alpha$, whose thermal speed is $v_{T \alpha}$ at temperature $T_{\alpha}$. Taking the radius of curvature within the middle magnetosphere current sheet to be $\sim 2 R_{J}$ (e.g. Mauk and Krimigis, 1987; Staines et al., 1996), and the ion and electron temperatures to be $\sim 100 \mathrm{eV}$ (e.g. Acuña et al., 1983; Belcher, 1983), then yields

$g_{i x}^{\text {cur }} \approx \frac{m_{e}}{m_{i}} g_{\text {ex }}^{\text {cur }} \approx \frac{T}{2 m_{i} R_{J}} \sim 4 \mathrm{~m} \mathrm{~s}^{-2}$,

where for definiteness we have taken the ion mass to correspond to oxygen. We note that particle guiding-centre motion is indeed valid for these parameters, since even for oxygen ions we typically find $\left(\rho_{i} / R_{J}\right) \approx 10^{-2}$ within the relevant region of the current sheet, where $\rho_{i}$ is the ion gyroradius, compared with current sheet scales of $\sim R_{J}$ or larger. It can thus be seen that for ions the curvature effect is generally small compared with the centrifugal force, and vice-versa for electrons.

We should comment at this point that the above development of the problem using straight field lines in a Cartesian geometry forms an approximation that significantly simplifies the algebra of the problem and allows a simple treatment of the physical effects of interest. Although this geometry may initially seem a rather poor representation of the jovian middle magnetosphere, we emphasise that the essential features of the "current sheet" form of the field lines are in fact appropriately included via the "external" forces representing the centrifugal effect of plasma rotation and the field line curvature. Similar approximations have been used in a number of related works cited in the introduction. The approximation is valid provided the unstable modes are of small spatial scale in the azimuthal ( $y$ ) direction, such that the discrete nature of the spectrum of azimuthal wave numbers in the real system does not play a significant role, and that the bulk of the plasma is confined to the vicinity of the equatorial plane, as we assume.

We should also comment on the neglect of the plasma pressure. Previous results e.g. due to Mikhailovskii (1974) have shown that pressure effects on the development of the instability can be neglected provided $\left(\rho_{i} / L\right)^{2} \ll L / R_{c}$, 
where $L$ is the radial scale length of plasma density variations, and $\rho_{i}$ and $R_{c}$ are the ion gyroradius and radius of curvature of the field lines, respectively, as above. We noted above that typically $\left(\rho_{i} / R_{J}\right) \approx 10^{-2}$ within the current sheet, so that this condition is very well satisfied for $L \sim R_{c} \sim R_{J}$ or larger.

With these explanations and justifications, the cold plasma equations for ions and electrons governing electrostatic perturbations of the system are therefore

$$
\begin{aligned}
& \frac{\partial \bar{n}_{\alpha}}{\partial t}+\operatorname{div}\left(\bar{n}_{\alpha} \overline{\boldsymbol{v}}_{\alpha}\right)=0, \\
& \bar{\rho}_{\alpha}\left(\frac{\partial \overline{\boldsymbol{v}}_{\alpha}}{\partial t}+\left(\overline{\boldsymbol{v}}_{\alpha} \cdot \nabla\right) \overline{\boldsymbol{v}}_{\alpha}\right)=q_{\alpha} \bar{n}_{\alpha}\left(\overline{\boldsymbol{E}}+\overline{\boldsymbol{v}}_{\alpha} \times \boldsymbol{B}\right)+\bar{\rho}_{\alpha} \boldsymbol{g}_{\alpha},
\end{aligned}
$$

$\operatorname{div} \overline{\boldsymbol{E}}=e\left(\bar{n}_{i}-\bar{n}_{e}\right) / \varepsilon_{o}$,

and

curl $\overline{\boldsymbol{E}}=\mathbf{0}$,

where $\alpha$ again denotes the particle species, with $\alpha=i$ corresponding to ions and $\alpha=e$ to electrons, whose charges are $q_{i}=-q_{e}=e$, the absolute value of the electronic charge. The quantities with bars over them are those which vary in the perturbed system, so that $\bar{n}_{\alpha}=n_{\alpha}^{(0)}+n_{\alpha}$ and $\overline{\boldsymbol{v}}_{\alpha}=\boldsymbol{v}_{\alpha}^{(0)}+\boldsymbol{v}_{\alpha}$ are the total density and velocity of species $\alpha$ respectively (zeroth order value plus perturbation), and $\bar{\rho}_{\alpha}=m_{\alpha} \bar{n}_{\alpha}$ is the corresponding mass density. The fields $\boldsymbol{B}=B(x) \hat{z}$ and $\boldsymbol{g}_{\alpha}=g_{\alpha}(x) \hat{\boldsymbol{x}}$ are the unvarying magnetic field and the "external" force per unit mass on the particles in the equatorial plasma slab, respectively, where the latter is in general given by the sum of terms in Eqs. (3) and (4). The electric field, however, is in general given by $\overline{\boldsymbol{E}}=\boldsymbol{E}^{(0)}-\nabla \varphi$, where $\boldsymbol{E}^{(0)}$ is the $x$-directed zeroth order electric field associated with the convection velocity given by Eq. (2) (such that $\left.\boldsymbol{E}^{(0)}=-V_{y} \hat{\boldsymbol{y}} \times \boldsymbol{B}\right)$, and $\varphi$ is the potential associated with the perturbation. It is convenient in the analysis below, however, to work in the local frame of reference in which $\boldsymbol{E}^{(0)}=\mathbf{0}$, corresponding to the frame which is locally convecting with the plasma in the $y$ direction. We note from Eq. (2) that the plasma is taken to have a fixed velocity $V=15 \Omega_{J} R_{J}$ at "radial" distances $x \geq 15 R_{J}$, to which the theory presented here is principally applied, in which case a single transformation removes the zeroth order electric field at all distances beyond $15 R_{J}$. If we then examine Eq. (5b) at zeroth order, we find that particles of species $\alpha$ drift in this frame under the action of the "external" force with a speed

$\boldsymbol{v}_{\alpha}^{(0)}=-\frac{m_{\alpha} g_{\alpha}(x)}{q_{\alpha} B(x)} \hat{\boldsymbol{y}}$

ions and electrons drifting in opposite directions. We also note that although the divergence of $\boldsymbol{E}^{(0)}$ associated with the sub-corotational convective flow may not generally be exactly equal to zero, its value is sufficiently small that we can take the zeroth order densities of ions and electrons to be equal in the perturbation analysis below, that is

$n_{i}^{(0)}=n_{e}^{(0)}=n^{(0)}$.

However, Eq. (7) is exactly satisfied in our transformed model system for distances beyond $x=15 R_{J}$, where $\boldsymbol{E}^{(0)}$ is simultaneously zero at all larger distances as noted above.

\section{Dispersion equation for electrostatic perturbations}

According to Mikhailovskii (1974), the flute perturbations that are the most unstable correspond to modes propagating in the $y-z$ plane with finite $k_{y}$ and $k_{z}$, but with zero "radial" wave number $k_{x}$. In this case the electric field of the wave, $\overline{\boldsymbol{E}}=-\nabla \varphi=i \boldsymbol{k} \varphi$, also lies in the $y-z$ plane, such that the $\boldsymbol{E} \times \boldsymbol{B}$ drift of the plasma associated with the interchange motions is directed wholly in the $x$ direction, perpendicular to the surfaces of constant density. Unstable modes with finite $k_{x}$ are found to have growth rates which are smaller by the factor $\sim k_{y}^{2} /\left(k_{x}^{2}+k_{y}^{2}\right)$. We thus consider perturbations of the form $\exp \left(i \omega t-i k_{y} y-i k_{z} z\right)$. Then putting $\bar{n}_{\alpha}=n_{\alpha}^{(0)}+n_{\alpha}$ and $\overline{\boldsymbol{v}}_{\alpha}=\boldsymbol{v}_{\alpha}^{(0)}+\boldsymbol{v}_{\alpha}$ in Eq. (5), and retaining first order terms only, yields the following. From the continuity equation Eq. (5a) we obtain the density perturbation as

$n_{\alpha}=\frac{i \operatorname{div}\left(n^{(0)} \boldsymbol{v}_{\alpha}\right)}{\omega_{\alpha}^{\prime}}$

where

$\omega_{\alpha}^{\prime}=\omega-k_{y} v_{\alpha y}^{(0)}$

is the Doppler-shifted wave frequency in the rest frame of species $\alpha$, and drift velocity $v_{\alpha y}^{(0)}$ is given by Eq. (6). Similarly the momentum equation Eq. (5b) gives at first order

$i \omega \boldsymbol{v}_{\alpha}+\left(\boldsymbol{v}_{\alpha} \cdot \nabla\right) \boldsymbol{v}_{\alpha}^{(0)}+\left(\boldsymbol{v}_{\alpha}^{(0)} \cdot \nabla\right) \boldsymbol{v}_{\alpha}=\frac{q_{\alpha}}{m_{\alpha}}\left(i \boldsymbol{k} \varphi+\boldsymbol{v}_{\alpha} \times \boldsymbol{B}\right)$,

which can be separated into a component parallel to the magnetic field, giving

$v_{\alpha z}=\frac{q_{\alpha}}{m_{\alpha}} \frac{k_{z} \varphi}{\omega_{\alpha}^{\prime}}$,

and a component which is perpendicular to the magnetic field

$$
\begin{gathered}
i \omega \boldsymbol{v}_{\alpha \perp}+\left(\boldsymbol{v}_{\alpha \perp} . \nabla\right) \boldsymbol{v}_{\alpha}^{(0)}+\left(\boldsymbol{v}_{\alpha}^{(0)} \cdot \nabla\right) \boldsymbol{v}_{\alpha \perp}= \\
\frac{q_{\alpha}}{m_{\alpha}}\left(i \boldsymbol{k}_{\perp} \varphi+\boldsymbol{v}_{\alpha \perp} \times \boldsymbol{B}\right),
\end{gathered}
$$

where $k_{\perp}=k_{y} \hat{\boldsymbol{y}}$. The second term on the LHS can be neglected since the scale length of variation of the zeroth order drift velocity in the $x$ direction is generally very large, such that solving for $\boldsymbol{v}_{\alpha \perp}$ we obtain 


$$
\begin{gathered}
\boldsymbol{v}_{\alpha \perp}=\frac{1}{\omega_{B \alpha}^{2}-\omega_{\alpha}^{\prime 2}} \frac{q_{\alpha}}{m_{\alpha}} \varphi\left(i \frac{q_{\alpha}}{m_{\alpha}} \boldsymbol{k}_{\perp} \times \boldsymbol{B}-\omega_{\alpha}^{\prime} \boldsymbol{k}_{\perp}\right)= \\
\frac{1}{\omega_{B \alpha}^{2}-\omega_{\alpha}^{\prime 2}} \frac{q_{\alpha}}{m_{\alpha}} k_{y} \varphi\left(i \omega_{B \alpha} \hat{\boldsymbol{x}}-\omega_{\alpha}^{\prime} \hat{\boldsymbol{y}}\right),
\end{gathered}
$$

where

$\omega_{B \alpha}=\frac{q_{\alpha} B}{m_{\alpha}}$

is the gyrofrequency of species $\alpha$. Substituting Eqs. (11) and (13) into Eq. (8) then yields

$$
n_{\alpha}=\frac{q_{\alpha}}{m_{\alpha}} \varphi\left(n^{(0)}\left(\frac{k_{z}^{2}}{\omega_{\alpha}^{\prime 2}}-\frac{k_{y}^{2}}{\omega_{B \alpha}^{2}-\omega_{\alpha}^{\prime 2}}\right)-\frac{k_{y}}{\omega_{\alpha}^{\prime}} \frac{\partial}{\partial x}\left(\frac{n^{(0)} \omega_{B \alpha}}{\omega_{B \alpha}^{2}-\omega_{\alpha}^{\prime 2}}\right)\right)
$$

We now substitute Eq. (15) for ions and electrons into Poisson's equation Eq. (5c) to obtain the general dispersion equation of the waves. Assuming

$$
\left|\omega_{B \alpha}\right| \gg\left|\omega_{\alpha}^{\prime}\right|
$$

for both species throughout Eq. (15), and employing Eqs. (6) and (9) in the final term, we obtain

$$
\begin{gathered}
k_{y}^{2}\left(1+\frac{\omega_{p i}^{2}}{\omega_{B i}^{2}}+\frac{\omega_{p e}^{2}}{\omega_{B e}^{2}}\right)+k_{z}^{2}\left(1-\frac{\omega_{p i}^{2}}{\omega_{i}^{\prime 2}}-\frac{\omega_{p e}^{2}}{\omega_{e}^{\prime 2}}\right)- \\
\frac{k_{y}^{2}}{\omega_{B i}}\left(\frac{g_{i x}+\left(m_{e} / m_{i}\right) g_{e x}}{\omega_{i}^{\prime} \omega_{e}^{\prime}}\right) \frac{\partial}{\partial x}\left(\frac{\omega_{p i}^{2}}{\omega_{B i}}\right)=0,
\end{gathered}
$$

where

$\omega_{p \alpha}=\sqrt{\frac{n^{(0)} e^{2}}{\varepsilon_{o} m_{\alpha}}}$

is the plasma frequency of species $\alpha$.

Equation (17) applies both to the equatorial plasma slab (region I), and to the more rarefied plasma between the slab and the ionosphere (region II). Let us estimate some typical values of the frequencies involved. At a radial distance of $\sim 15 R_{J}$ within the equatorial plasma sheet, for example, we have $B \sim 50 \mathrm{nT}$ and $n_{I}^{(0)} \sim 10 \mathrm{~cm}^{-3}$ (e.g.Ãcuña et al., 1983; Belcher, 1983), so that $\omega_{p i I}^{2} / \omega_{B i}^{2} \sim 10^{7}$. Thus within the first term of Eq. (17) we find $\omega_{\text {pi I }}^{2} / \omega_{B i}^{2} \gg \omega_{\text {peI }}^{2} / \omega_{B e}^{2} \sim 1$. In the second term we also have $\omega_{p e I}^{2} / \omega_{e}^{\prime 2} \gg \omega_{p i I}^{2} / \omega_{i}^{\prime 2} \gg 1$. In the third term we note from Eqs. (3) and (4) that the dominant term due to the "external" force corresponds to the centrifugal force on the ions, whose value, outside of $15 R_{J}$, is given by Eq. (3b). Thus including only the dominant components in each term, in region I Eq. (17) becomes

$k_{y}^{2}\left(\frac{\omega_{p i I}^{2}}{\omega_{B i}^{2}}\right)-k_{z}^{2}\left(\frac{\omega_{p e I}^{2}}{\omega_{e}^{\prime 2}}\right)-\frac{k_{y}^{2}}{\omega_{B i}}\left(\frac{V^{2}}{x \omega_{i}^{\prime} \omega_{e}^{\prime}}\right) \frac{\partial}{\partial x}\left(\frac{\omega_{p i I}^{2}}{\omega_{B i}}\right)=0$, where, as in Eq. (2), $V=15 \Omega_{J} R_{J}$. In region II we also ignore the effect of the "external" force, as indicated above, such that $\omega_{i}^{\prime}=\omega_{e}^{\prime}=\omega$, and we find

$\omega^{2}=\frac{m_{i}}{m_{e}} \frac{k_{z}^{2}}{k_{y}^{2}} \omega_{B i}^{2}=\frac{k_{z}^{2}}{k_{y}^{2}} \omega_{L H}^{2}$,

where $\omega_{L H}=\sqrt{\omega_{B i}\left|\omega_{B e}\right|}$ is the lower hybrid frequency.

\section{Boundary conditions and parallel wave numbers}

Equations (19) and (20) do not represent a complete solution to the problem, since the parallel wave number $k_{z}$, in particular, remains undetermined. Here we determine $k_{z}$ by consideration of the boundary conditions at the edge of the slab and in the ionosphere. These boundary conditions follow from Faraday's law, which requires the electric field parallel to the boundaries to be continuous across them, and from the requirement for charge conservation. First, however, we consider the nature of the solutions we are seeking.

\subsection{Form of the potential perturbations}

Since the system is bounded along the magnetic field in the $z$ direction, we consider perturbations consisting of the sum of two waves of the same angular frequency $\omega$ and perpendicular wave number $k_{y}$, but opposite parallel wave number $k_{z}$. The electric potential perturbation in region $I$ is thus written as

$$
\begin{aligned}
\varphi_{I}= & \varphi_{I}^{+}+\varphi_{I}^{-}=\Phi_{I}^{+} \exp \left(i \omega_{I} t-i k_{I y} y-i k_{I z} z\right)+ \\
& \Phi_{I}^{-} \exp \left(i \omega_{I} t-i k_{I y} y+i k_{I z} z\right)
\end{aligned}
$$

where $\Phi_{I}^{+}$and $\Phi_{I}^{-}$are the corresponding plane wave amplitudes. On the basis of the results of Bespalov and Davydenko (1994), who considered the flute instability of a plasma disk in the case where ionospheric effects are neglected, we may expect that the most unstable mode corresponds to the lowest mode which is even in $z$, such that the field-aligned electric field goes to zero at the centre of the disk. In this case we only consider one half of the system, say for $z$ positive, with the boundary condition $\partial \varphi_{I} / \partial z=0$ at $z=0$. From Eq. (21) this gives $\Phi_{I}^{+}=\Phi_{I}^{-}=\Phi_{I}$, so that the disturbance in region I can be written as

$\varphi_{I}=2 \Phi_{I} \exp \left(i \omega_{I} t-i k_{I y} y\right) \cos \left(k_{I z} z\right)$.

In region II, however, we use the full expression

$$
\begin{aligned}
\varphi_{I I}= & \varphi_{I I}^{+}+\varphi_{I I}^{-}=\Phi_{I I}^{+} \exp \left(i \omega_{I I} t-i k_{I I y} y-i k_{I I z} z\right)+ \\
& \Phi_{I I}^{-} \exp \left(i \omega_{I I} t-i k_{I I y} y+i k_{I I z} z\right)
\end{aligned}
$$

where we initially assume for generality that the angular frequencies and wave numbers are different in the two regions. 
4.2 Boundary conditions at the interface between regions I and II

We first consider the boundary conditions at the edge of the equatorial plasma slab at $z=d$, at the interface between regions I and II. Faraday's law (Eq. 5d) firstly requires that the electric field parallel to the boundary, in this case the $y$ component, be continuous across it. Differentiating Eqs. (22) and (23) then gives

$$
\begin{gathered}
2 k_{I y} \Phi_{I} \exp \left(i \omega_{I} t-i k_{I y} y\right) \cos \left(k_{I z} d\right)= \\
k_{I I y} \Phi_{I I}^{+} \exp \left(i \omega_{I I} t-i k_{I I y} y-i k_{I I z} d\right)+ \\
\Phi_{I I}^{-} \exp \left(i \omega_{I I} t-i k_{I I y} y+i k_{I I z} d\right),
\end{gathered}
$$

which must be satisfied for all $y$ and $t$. This firstly requires that the angular frequency $\omega$ and the perpendicular wave number $k_{y}$ must have the same values in the two regions, so that

$\omega_{I}=\omega_{I I}=\omega \quad k_{I y}=k_{I I y}=k_{y}$.

We then also note from the dispersion equation in region II (Eq. 20) that

$k_{I I z}=\frac{\omega}{\omega_{L H}} k_{y}$.

Substituting Eqs. (25) and (26) into Eq. (24) then gives

$$
\begin{gathered}
2 \Phi_{I} \cos \left(k_{I z} d\right)=\Phi_{I I}^{+} \exp \left(-i \frac{\omega}{\omega_{L H}} k_{y} d\right)+ \\
\Phi_{I I}^{-} \exp \left(i \frac{\omega}{\omega_{L H}} k_{y} d\right) .
\end{gathered}
$$

The second boundary condition at $z=d$ is that of charge conservation. From Ampère's law we have $\operatorname{div}\left(\boldsymbol{j}+\varepsilon_{o} \partial \boldsymbol{E} / \partial t\right)=0$ (consistent with Eqs. 5a and 5c), which applied to the perturbation at the boundary gives $j_{z}+i \varepsilon_{o} \omega E_{z}=$ const. To first order, the current density in the $z$ direction is given by

$j_{z}=\sum_{\alpha} q_{\alpha} n^{(0)} v_{\alpha z}$

where $v_{\alpha z}$ is given by Eq. (11) for a single plane wave mode. Applying this in region I to the pair of modes represented by Eq. (22) yields

$j_{I z}=-2 i \frac{e^{2} n_{I}^{(0)} k_{I z}}{m_{e} \omega} \Phi_{I} \sin \left(k_{I z} z\right) \exp \left(i \omega t-i k_{y} y\right)$,

where only the dominant electron term has been retained, in which the small Doppler shift term has been neglected in the expression for the angular frequency in the denominator. Similarly, using the pair of modes given by Eq. (23) in region II we have

$$
\begin{aligned}
& j_{I I z}=\frac{e^{2} n_{I I}^{(0)} k_{y}}{m_{e} \omega} \frac{\omega}{\omega_{L H}} \times \\
& \left(\Phi_{I I}^{+} \exp \left(-i \frac{\omega}{\omega_{L H}} k_{y} z\right)-\Phi_{I I}^{-} \exp \left(i \frac{\omega}{\omega_{L H}} k_{y} z\right)\right) \exp \left(i \omega t-i k_{y} y\right) .
\end{aligned}
$$

We then find from Eqs. (29) and (30) that the ratio of the two terms in the continuity equation is $\left|\varepsilon_{o} \omega E_{z} / j_{z}\right|=\left(\omega / \omega_{p e}\right)^{2} \gg 1$, where $\omega_{p e}$ is the electron plasma frequency in either region. (We note that the implication that curl $\boldsymbol{B}$, and hence the magnetic field of the perturbation, is not strictly zero does not invalidate the electrostatic approximation employed here, provided that the wave phase speed is much less than the speed of light. In this case $\boldsymbol{E}$ and $\boldsymbol{k}$ are almost parallel for a plane wave, if not exactly so.) With the above inequality, the conservation condition at the boundary thus reduces essentially to continuity of the fieldaligned current density. Equating Eqs. (29) and (30) at $z=d$ then yields

$$
\begin{aligned}
& -2 i n_{I}^{(0)} k_{I z} \Phi_{I} \sin \left(k_{I z} d\right)= \\
& n_{I I}^{(0)} k_{y} \frac{\omega}{\omega_{L H}}\left(\Phi_{I I}^{+} \exp \left(-i \frac{\omega}{\omega_{L H}} k_{y} d\right)-\Phi_{I I}^{-} \exp \left(i \frac{\omega}{\omega_{L H}} k_{y} d\right)\right),
\end{aligned}
$$

which gives a second relationship between the amplitudes $\Phi_{I}, \Phi_{I I}^{+}$, and $\Phi_{I I}^{-}$, additional to Eq. (27) obtained from Faraday's law at the boundary. If we then divide Eq. (31) by Eq. (27) we can eliminate $\Phi_{I}$ to obtain

$$
\begin{aligned}
& k_{I z} \tan \left(k_{I z} d\right)= \\
& i \tau k_{y} \frac{\omega}{\omega_{L H}} \frac{\left(1-\frac{\Phi_{I I}^{-}}{\Phi_{I I}^{+}} \exp \left(2 i \frac{\omega}{\omega_{L H}} k_{y} d\right)\right)}{\left(1+\frac{\Phi_{I I}^{-}}{\Phi_{I I}^{+}} \exp \left(2 i \frac{\omega}{\omega_{L H}} k_{y} d\right)\right)},
\end{aligned}
$$

where $\tau<1$ is the ratio of the plasma densities outside and inside the equatorial plasma slab, as in Eq. (1).

\subsection{Boundary conditions at the ionosphere}

In order to determine the relationship between $\Phi_{I I}^{+}$and $\Phi_{I I}^{-}$ in Eq. (32) we now examine the boundary condition at the ionosphere, at $z=l$. One important feature of the real system compared with the straight field line approximation employed here, is that the field lines strongly converge as they approach the ionosphere, so that the cross-field spatial scale of the perturbations also decrease. An equatorial azimuthal segment of angular width $d \varphi$ at radius $L R_{J}$ has a length $L R_{J} d \varphi$, while mapped along the field lines to the ionosphere the corresponding azimuthal length for a dipole field is $R_{J} d \varphi / \sqrt{L}$. Consequently the cross-field wave numbers in the ionosphere and in the equatorial magnetosphere are related by $k_{y}^{i o n}=k_{y} L^{3 / 2}$.

From Faraday's law, the $y$-directed electric field within the ionosphere is equal to that in the magnetospheric plasma just outside conducting layer, so that the height-integrated ionospheric Pedersen current driven by the electric field of the wave is

$$
i_{y}=\Sigma_{P} E_{y}^{i o n}=i \Sigma_{P} k_{y}^{i o n}\left(\varphi_{I I}^{+}(z=l)+\varphi_{I I}^{-}(z=l)\right) .
$$


Charge conservation at $z=l$ again reduces essentially to continuity of the field-aligned current density, such that

$$
\begin{gathered}
j_{I I z}^{i o n}(z=l)=\frac{\partial i_{y}}{\partial y}=-i k_{y}^{i o n} i_{y}= \\
\Sigma_{P}\left(k_{y}^{i o n}\right)^{2}\left(\varphi_{I I}^{+}(z=l)+\varphi_{I I}^{-}(z=l)\right) \approx \\
L^{3} \Sigma_{P} k_{y}^{2}\left(\varphi_{I I}^{+}(z=l)+\varphi_{I I}^{-}(z=l)\right),
\end{gathered}
$$

where $j_{I I z}^{i o n}(z=l)$ is the field-aligned current density of the wave at the ionosphere, as also modified by field line convergence. To account for the latter we simply use the condition that $j_{z} / B$ is a constant, so that $j_{I I z}^{\text {ion }}(z=l) \approx L^{3} j_{I I z}(z=l)$, where $j_{I I z}(z=l)$ is the value given by Eq. (30). Thus from Eq. (30) we have

$j_{I I z}^{i o n}(z=l) \approx L^{3} \frac{\varepsilon_{o} \omega_{p e I I}^{2} k_{y}}{\omega_{L H}}\left(\varphi_{I I}^{+}(z=l)-\varphi_{I I}^{-}(z=l)\right)$,

so that substitution into Eq. (34) and rearranging gives

$\left(\frac{\omega_{p e I I}^{2}}{\omega_{L H}}+\frac{\Sigma_{P} k_{y}}{\varepsilon_{o}}\right) \varphi_{I I}^{-}(z=l)=\left(\frac{\omega_{p e I I}^{2}}{\omega_{L H}}-\frac{\Sigma_{P} k_{y}}{\varepsilon_{o}}\right) \varphi_{I I}^{+}(z=l)$.

Substituting for the potential functions from Eq. (23) finally gives

$\Phi_{I I}^{-}=\Phi_{I I}^{+} A\left(k_{y}\right) \exp \left(-2 i \frac{\omega}{\omega_{L H}} k_{y} l\right)$

where

$A\left(k_{y}\right)=\frac{\left(\frac{\omega_{p e I I}^{2}}{\omega_{L H}}-\frac{\Sigma_{P} k_{y}}{\varepsilon_{o}}\right)}{\left(\frac{\omega_{p e I I}^{2}}{\omega_{L H}}+\frac{\Sigma_{P} k_{y}}{\varepsilon_{o}}\right)}$.

Substituting Eq. (37) into Eq. (32) then yields the following equation for the region I parallel wave number $k_{I z}$ for given $\omega$ and $k_{y}$

$$
\begin{aligned}
& k_{I z} \tan \left(k_{I z} d\right)= \\
& \quad i \tau k_{y} \frac{\omega}{\omega_{L H}} \frac{\left(1-A\left(k_{y}\right) \exp \left(-2 i \frac{\omega}{\omega_{L H}} k_{y}(l-d)\right)\right)}{\left(1+A\left(k_{y}\right) \exp \left(-2 i \frac{\omega}{\omega_{L H}} k_{y}(l-d)\right)\right)} .
\end{aligned}
$$

This, together with the dispersion relation in region I given by Eq. (19), then describes the flute instability of the plasma disk.

\section{Threshold profile of the plasma density in the equa- torial disk}

If a strong radial gradient of the plasma density exists within the plasma disk, such that a flute instability develops, then the resulting radial plasma transport will be such as to reduce the density gradient so that near-stability is restored.
Here we therefore determine the radial profile of the plasma density such that it lies at the threshold of flute instability at all distances. This profile then represents the steepest that can exist which is just stable to flute perturbations.

We first consider in more detail the restrictions placed on the "azimuthal" wave number $k_{y}$, and the consequences that follow. As indicated above, a principal requirement for the validity of our Cartesian slab model is that $\left|k_{y}\right|$ should be sufficiently large that the actual discreteness of the spectrum of azimuthal wave numbers can be neglected. We thus require that the azimuthal wavelength be much smaller than the circumference at a given radius, that is

$\left|k_{y}\right| \gg \frac{1}{x}$

If, however, $\left|k_{y}\right|$ also satisfies

$\left|k_{y}\right| \gg \frac{\varepsilon_{o} \omega_{p e I I}^{2}}{\Sigma_{P} \omega_{L H}}$

then we find from Eq. (38) that $A\left(k_{y}\right)=-1$. This is the limit in which the ionosphere behaves as a perfect conductor, such that it represents a node of the transverse wave electric field $E_{y}$. In this case Eq. (39) then becomes

$k_{I z} \tan \left(k_{I z} d\right)=i \tau k_{y} \frac{\omega}{\omega_{L H}} \frac{\left(1+\exp \left(-2 i \frac{\omega}{\omega_{L H}} k_{y}(l-d)\right)\right)}{\left(1-\exp \left(-2 i \frac{\omega}{\omega_{L H}} k_{y}(l-d)\right)\right)}$.

Now it is also known that the most unstable modes correspond to the smallest longitudinal wave numbers (Mikhailovskii, 1974), such that we look for solutions which satisfy

$k_{I z} d \ll 1$.

An analysis of the flute instability under the condition $k_{I z} d=0$ also shows that the unstable modes have very low real frequencies, so that we also suppose

$\frac{\omega}{\omega_{L H}}\left|k_{y}\right|(l-d) \ll 1$

Expansion of the trigonometric functions in Eq. (42) with use of Eq. (44) then yields simply

$k_{I z}^{2}=\frac{\tau}{d(l-d)}$.

Substitution of Eq. (45) into the dispersion equation for region I given by Eq. (19) then yields

$\omega_{i}^{\prime} \omega^{2}-\frac{\omega V^{2}}{x} \frac{\partial}{\partial x}\left(\ln \left(\frac{n_{I}^{(0)}}{B}\right)\right)-\omega_{i}^{\prime}\left(\frac{\tau \omega_{L H}^{2}}{d(l-d) k_{y}^{2}}\right)=0$, 


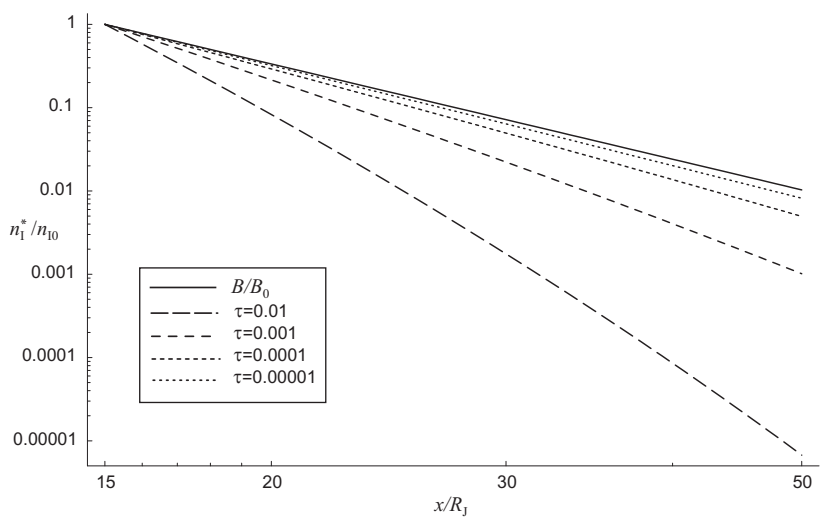

Fig. 2. Log-log plot of the "radial" profiles of the magnetic field strength $B$ (solid line), and the limiting plasma density within the equatorial plasma slab $n_{I}^{*}$ corresponding to the threshold of the flute instability, determined from Eq. (49) for various values of $\tau$ (dashed lines as indicated). The parameters are shown in the "radial" range $15 \leq\left(x / R_{J}\right) \leq 50$, corresponding to the Jovian middle magnetosphere, with values normalised to those at the inner edge at $\left(x / R_{J}\right)=15$ ( $B_{0}$ and $n_{I 0}$ respectively). From upper to lower, the dashed lines show the limiting density profiles for $\tau$ (the ratio of the plasma density inside and outside the plasma disk) equal to $10^{-5}$, $10^{-4}, 10^{-3}$, and $10^{-2}$, respectively.

where the Doppler-shifted frequency for ions is, from Eqs. (2), (3b), (6), and (9)

$\omega_{i}^{\prime}=\omega+\frac{k_{y} V^{2}}{\omega_{B i} x}$,

and where we have again neglected the small Doppler shift for electrons, such that $\omega_{e}^{\prime} \approx \omega$. We note that if we put $\tau=0$ in Eq. (46) then we recover the flute mode dispersion relation derived by Bespalov and Davydenko (1994) in the limit that the medium surrounding the equatorial plasma disk is treated as a vacuum.

Substitution of Eq. (47) into Eq. (46) yields a dispersion equation which is a cubic equation for the angular frequency $\omega$ at given $k_{y}$

$\omega^{3}+a \omega^{2}+b \omega+c=0$

where

$a=\frac{k_{y} V^{2}}{\omega_{B i} x}, \quad b=-\frac{V^{2}}{x} \frac{\partial}{\partial x}\left(\ln \left(\frac{n_{I}^{(0)}}{B}\right)\right)-\left(\frac{\tau \omega_{L H}^{2}}{d(l-d) k_{y}^{2}}\right)$,

and

$c=-\frac{k_{y} V^{2}}{\omega_{B i} x}\left(\frac{\tau \omega_{L H}^{2}}{d(l-d) k_{y}^{2}}\right)$.

The solutions of Eq. (48) consist either of three real roots, or one real root and a complex conjugate pair, one of which corresponds to instability. The transition between these solution types, corresponding to the threshold of the flute instability, occurs when the discriminant $D$ of the cubic equation is zero, that is

$D=\frac{\left(b-\left(a^{2} / 3\right)\right)^{3}}{27}+\frac{\left(\left(2 a^{3} / 27\right)-(a b / 3)+c\right)^{2}}{4}=0$,

The condition for three real roots is $D \leq 0$. As can be seen, at a given position $x$ Eq. (49) is itself a cubic equation for the gradient $\partial\left(\ln \left(n_{I}^{(0)} / B\right)\right) / \partial x$ for given $k_{y}$, from which the limiting density profile for given $B(x)$ can be calculated numerically, as we now discuss.

To undertake these numerical calculations, we must first choose suitable representative values for the model parameters, together with the magnetic field profile. In the calculations presented here we have taken the half-width of the plasma disk $d=2 R_{J}$, the length of the field lines $l=1.3 x$, and (as above) the "azimuthal" velocity of the plasma $V=15 \Omega_{J} R_{J} \approx 190 \mathrm{~km} \mathrm{~s}^{-1}$. Results will be presented for several values of the density ratio $\tau<1$. For the magnetic field model we have taken a simple power law $B(x) \propto x^{-\beta}$, where specifically $\beta=3.8$. As we will see below, this corresponds to a typical behaviour of the equatorial magnetic field in the inner part of the middle magnetosphere, over a radial range from $\sim 10$ to $\sim 30 R_{J}$. At larger distances the equatorial field falls less steeply with distance (Khurana and Kivelson, 1993). Numerical analysis then shows that the discriminant of the cubic equation given by Eq. (49) is positive, such that there is only one real solution for $\partial\left(\ln \left(n_{I}^{(0)} / B\right)\right) / \partial x$ at a given $k_{y}$. This root is always negative (such that $n_{I}^{(0)}$ decreases with $x$ faster than $B$ ), and at a given $x$ has a single (negative) maximum value which we denote as $\partial\left(\ln \left(n_{I}^{*} / B\right)\right) / \partial x$, at a wave number which we denote as $k_{y}^{*}$. For $\partial\left(\ln \left(n_{I}^{(0)} / B\right)\right) / \partial x<\partial\left(\ln \left(n_{I}^{*} / B\right)\right) / \partial x$ we thus have a range of unstable modes, while for $\partial\left(\ln \left(n_{I}^{(0)} / B\right)\right) / \partial x>\partial\left(\ln \left(n_{I}^{*} / B\right)\right) / \partial x$ there are no unstable modes. The value of $\partial\left(\ln \left(n_{I}^{*} / B\right)\right) / \partial x$ at a given $x$ thus gives the steepest density profile that is just stable to the flute mode, while $k_{y}^{*}$ gives the flute mode wave number at this threshold of instability. The corresponding real frequency of the perturbation $\omega^{*}$ at the threshold of instability can then be determined by substitution of these values into Eq. (48). It should be noted that the values $\omega^{*}$ and $k_{y}^{*}$ must satisfy the inequalities given by Eqs. (16) and (44). It is found that these are satisfied only if $\tau<0.1$, i.e. if the plasma density outside the equatorial disk is sufficiently small compared with the disk density. The case for $\tau>0.1$ where these approximations are not appropriate will be discussed elsewhere.

Numerically calculated limiting density profiles determined by integrating the $\partial\left(\ln \left(n_{I}^{*} / B\right)\right) / \partial x$ values are plotted versus $\left(x / R_{J}\right)$ in Fig. 2 in $\log$-log format. The solid line shows the assumed magnetic field profile as given above, 
while the dashed lines show the density profiles for four values of $\tau$, namely $10^{-2}, 10^{-3}, 10^{-4}$ and $10^{-5}$, as indicated in the figure. The solutions are shown in the range of $\left(x / R_{J}\right)$ from 15 to 50 , and the parameter values are normalised to the values at $\left(x / R_{J}\right)=15\left(B_{0}\right.$ and $n_{I 0}$ respectively). It can be seen that for the smallest value, $\tau=10^{-5}$, the limiting density profile is close to the magnetic field profile, though falling slightly more rapidly with increasing distance (i.e. $\partial\left(\ln \left(n_{I}^{*} / B\right)\right) / \partial x$ is negative but small). This is in accordance with previous results which show that for the case of a vacuum outside the plasma disk, such that the influence of the coupling with the ionosphere is eliminated, marginal stability simply requires $\partial\left(\ln \left(n_{I}^{*} / B\right)\right) / \partial x=0$, such that $\left(n_{I}^{*} / B\right)$ is a constant. For very small $\tau$, therefore, we find that $n_{I}^{*}$ very nearly follows the magnetic field profile. As $\tau$ increases, however, the limiting profiles shown in Fig. 2 become increasingly steep, due physically to current feedback from the conducting ionosphere which damps the instability, and which allows stability with larger radial density gradients. The log-log profiles of the plasma density remain almost linear, however, indicating an approximate power law behaviour $n_{I}^{*} \propto x^{-\eta}$. For the cases shown, we find approximately that $\eta \sim 3.94$ for $\tau=10^{-5}$, corresponding closely to the chosen magnetic field exponent of 3.8 , increasing to $\sim 4.24$ for $\tau=10^{-4}, \sim 5.20$ for $\tau=10^{-3}$, and to $\sim 8.22$ for $\tau=10^{-2}$. The corresponding values of $k_{y}^{*}$, normalised to $R_{J}^{-1}$, are shown versus $\left(x / R_{J}\right)$ for the same $\tau$ values in Fig. 3. It can be seen that the values correspond to "azimuthal" wavelengths which are generally very small compared with the circumference around the planet at a given "radius", in conformity with the above assumptions. The corresponding real frequency of the perturbations, $\omega^{*}$, is found to be very low, and typically lies in the interval between $\sim 10^{-4}$ and $\sim 10^{-3} \mathrm{rad} \mathrm{s}^{-1}$.

\section{Comparison with observations}

It is of interest to make an initial comparison of these results with observed radial distributions of plasma in the jovian equatorial plasma disk, using density data from the Galileo spacecraft. Frank et al. (2002) have provided convenient power law fits to the density values observed within the equatorial current sheet on Galileo orbit G08 (4 May to 22 June 1997). Data were obtained between a periapsis of $\sim 10 R_{J}$ and an apoapsis of $\sim 100 R_{J}$, where the latter lay in the postmidnight sector of the magnetotail. It was found that the equatorial density falls very steeply with radial distance in the inner part of the system, but much less rapidly further out. For radial distances less than $20 R_{J}$ they found

$n_{e q}(r) \approx 3.2 \times 10^{8}\left(\frac{R_{J}}{r}\right)^{6.9} \mathrm{~cm}^{-3}$

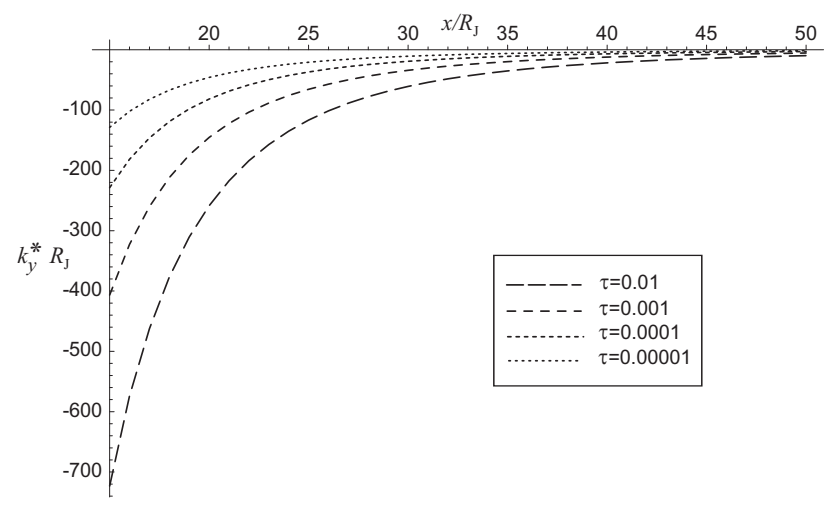

Fig. 3. Plot of the $y$ component of the flute mode wave vector (the "azimuthal" wave number), normalised to $R_{J}^{-1}$, corresponding to the threshold of instability at the limiting density gradient shown in Fig. 2, plotted versus "radial" distance $x$ for various values of $\tau$. From upper to lower, the dashed lines thus show $k_{y}^{*} R_{J}$ versus $\left(x / R_{J}\right)$ for $\tau$ equal to $10^{-5}, 10^{-4}, 10^{-3}$, and $10^{-2}$, respectively, as indicated.

while for radial distances beyond $50 R_{J}$ they obtained

$n_{e q}(r) \approx 9.8\left(\frac{R_{J}}{r}\right)^{1.28} \mathrm{~cm}^{-3}$.

Between $\sim 20$ and $\sim 50 R_{J}$ the density data are relatively sparse, but can reasonably be represented by an overall profile given by the sum of the above two profiles. This is shown in $\log$-log format in Fig. 4a, where the solid line shows the sum of the above two functions, while the two dashed straight lines show the power laws to which this asymptotes at small and large radial distances as given by the fits to the data. Results are shown over the radial range from 10 to $60 R_{J}$, thus overlapping the range of the theoretical results shown in Figs. 2 and 3.

The theoretical results derived in Sect. 5 above and shown in Fig. 2 concern the radial variation of the quantity $(n / B)$ in the equatorial plasma disk. We thus need to compare the observed density profile given in Eq. (50) with that of the magnetic field strength which threads through the disk in the equatorial plane. For this purpose we have obtained the G08 magnetic field data from the Planetary Data System at UCLA, and have derived a profile that describes the field strength minima as the spacecraft passes through the centre of the middle magnetosphere current sheet twice per planetary rotation. The form of this field strength profile follows that employed in the theoretical study of Nichols and Cowley (2004), which was based on the Voyager results of Connerney et al. (1981) for distances within $\sim 20 R_{J}$, and on the results of Khurana and Kivelson (1993) at distances beyond. The Nichols and Cowley (2004) profile has then been slightly modified to provide an improved fit to the G08 field minima 


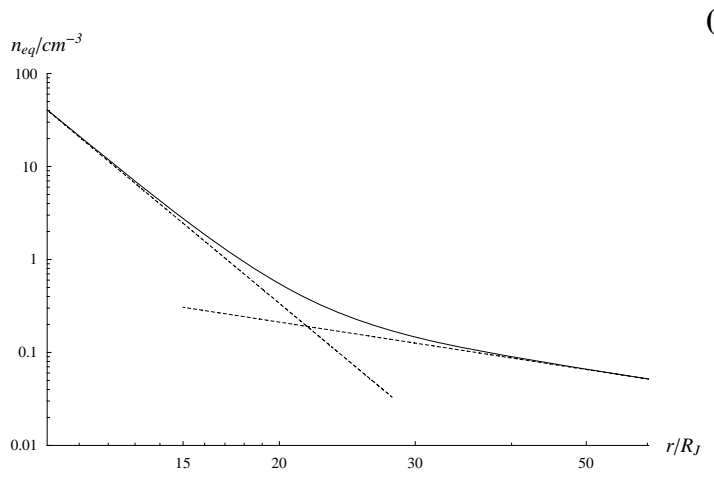

(a)

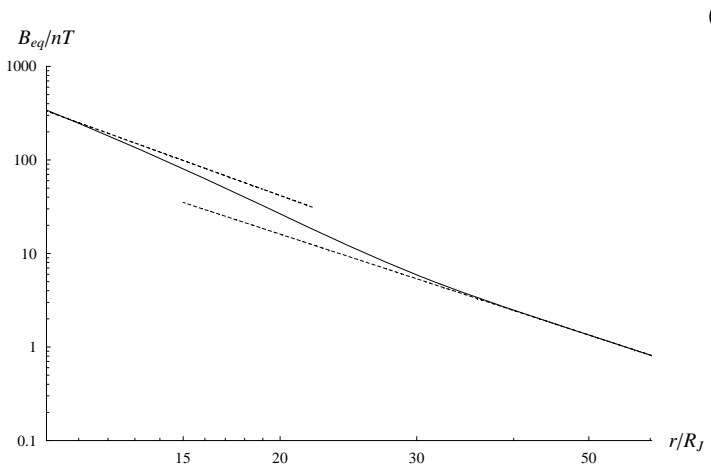

(b)

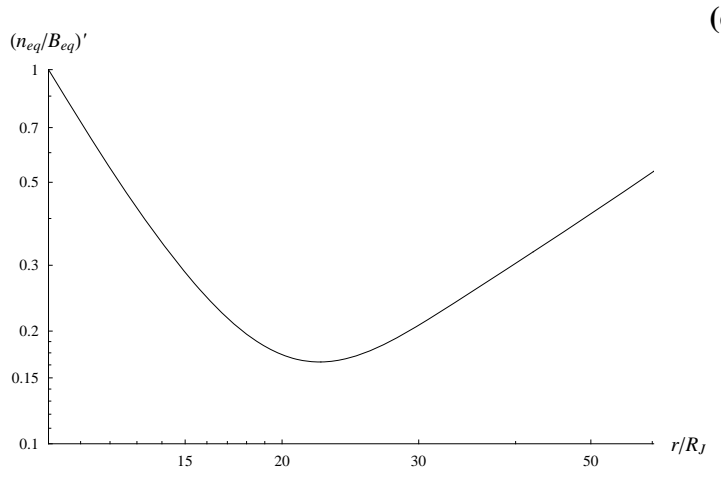

Fig. 4. Equatorial thermal plasma density and magnetic field strength profiles are shown plotted versus radial distance from Jupiter in the range from 10 to $60 R_{J}$, as determined from data obtained on Galileo orbit G08. The dashed straight lines in plot (a) show the power-law fits to the equatorial thermal plasma density values $n_{e q}$ in the radial ranges for $r<20 R_{J}$ and $r>50 R_{J}$, respectively, determined by Frank et al. (2002) and given by Eq. (50). The solid line shows their sum, taken to represent the overall density profile in the radial range shown. The solid line in plot (b) shows the profile of equatorial field strength minima $B_{e q}$ on the orbit, again in log-log format, represented by Eq. (52). The lower dashed line shows the second term only in Eq. (52), corresponding to the Khurana and Kivelson (1993) model. The upper dashed line shows the first term in Eq. (52) with the exponential term set to unity, such that the value varies with distance as the inverse cube. Plot (c) shows a log-log plot of the ratio $\left(n_{e q} / B_{e q}\right)$, normalised to its value at $10 R_{J}$. in the radial range between $\sim 15$ and $\sim 20 R_{J}$. The resulting field strength model is given by

$B_{e q}(r)=B_{o}\left(\frac{R_{J}}{r}\right)^{3} \exp \left[-\left(\frac{r}{r_{o}}\right)^{2}\right]+A\left(\frac{R_{J}}{r}\right)^{m}$,

where $B_{o}=3.335 \times 10^{5} \mathrm{nT}, r_{o}=17 R_{J}, A=5.4 \times 10^{4} \mathrm{nT}$ and $m=2.71$. The second term in this expression is the Khurana and Kivelson (1993) model, while the first is a modified dipole in form. Direct comparison with the G08 data shows that Eq. (52) provides a good description of the field strength minima out to at least $\sim 60 R_{J}$, for which purpose it is employed here. A log-log plot of the field profile is shown by the solid line in Fig. 4b. The lower dashed line shows the second term in Eq. (52) alone (the Khurana and Kivelson (1993) model), to which the solid curve asymptotes at distances beyond $\sim 35 R_{J}$. The upper dashed line shows the first term in Eq. (52) alone with the exponential set equal to unity, which thus decreases with distance as the inverse cube. It can thus be seen that over the radial range from $\sim 10$ to $\sim 30 R_{J}$ the equatorial field falls somewhat more steeply than for a dipole field, investigation of the profile showing that a power law of $\sim r^{-3.8}$ provides a good description.

We thus find that at radial distances between $\sim 10$ and $\sim 20 R_{J}$, the equatorial field strength on orbit G08 falls as $\sim r^{-3.8}$, while the plasma density determined by Frank et al. (2002) falls much more steeply as $\sim r^{-6.9}$. This density profile can then be compared directly with the theoretical results derived in Section 5 and illustrated in Fig. 2, since an equatorial magnetic field varying as $x^{-3.8}$ was specifically employed to derive those results, as noted above. Comparison with the results in Fig. 2 then implies that the ratio of the plasma density outside and inside the current sheet is approximately $\tau \approx 0.005$, a value which is in reasonable agreement e.g. with the Voyager results presented by Belcher (1983). We emphasise that such steep density profiles can exist in near-equilibrium only because of the electrical coupling of the plasma disk and the ionosphere, through the plasma that lies between. In making this simple comparison of equatorial plasma density and field strength profiles we are assuming, of course, that the plasma disk remains roughly constant in thickness over the radial range considered. However, modest variations in thickness will not change the nature of our conclusion. We are also assuming that the observed density profile is indeed close to the condition for marginal stability. In practice, the observed gradient might possibly represent conditions a little beyond this condition, such that slow interchange motions are in progress. In this case the value of $\tau$ might be a little lower than that estimated above.

Beyond $\sim 20 R_{J}$, however, the density profile in Fig. $4 \mathrm{a}$ falls more slowly than the field profile shown in Fig. $4 \mathrm{~b}$. This is shown explicitly in Fig. 4c, where we present the corresponding plot of $\left(n_{e q} / B_{e q}\right)$, normalised to the value at $10 R_{J}$. In the inner region this falls rapidly with distance as just described, reaches a minimum just beyond $\sim 20 R_{J}$, and 
then increases again at larger distances. The latter increase may in part be due to a thinning of the plasma sheet with increasing distance, resulting in an increase in the equatorial density for fixed plasma disk content. Increasing values of $\left(n_{e q} / B_{e q}\right)$ over and above this effect are clearly not expected on the basis of the theory presented here. As pointed out in the Introduction, however, the density profile can be further smoothed beyond the threshold profile calculated here by slower instabilities, to which we may then attribute the effect found here at larger distances.

\section{Summary}

In this paper we have examined the flute instability of the equatorial disk of iogenic plasma in Jupiter's middle magnetosphere. Particular attention has been paid in the analysis to the wave coupling between the dense plasma in the equatorial disk and the more rarefied plasma at higher latitudes, and between the latter plasma and the conducting planetary ionosphere. The analysis has been undertaken using a system with local Cartestian symmetry to simplify the geometric aspects of the problem, with "external" forces being used to represent the acceleration of the disk plasma due to plasma rotation and to field line curvature. The local Cartesian approximation is valid in the limit that the flute perturbations are of small spatial scale in the "azimuthal" direction. For such small-scale perturbations the ionosphere can also be treated as a perfect electrical conductor, and the condition under which this approximation holds has been determined.

We have then examined the conditions under which flute perturbations in this system are at the threshold of instability, and have used this to determine the corresponding limiting radial gradient of the plasma density within the plasma disk. In common with previous analyses, we find that when the density of the high-latitude plasma falls to values which are sufficiently low compared with the equatorial disk, such that coupling to the ionosphere becomes insignificant, the limiting density distribution follows that of the magnetic field, such that $(n / B)$ is a constant within the disk. However, as the density of the high-latitude plasma increases towards that of the disk, and with it the significance of the stabilising interaction with the ionosphere, then the limiting plasma density distribution in the disk falls increasingly steeply compared with that of the magnetic field.

Initial comparison with density values obtained by the Galileo spacecraft, specifically with those observed on orbit G08 presented by Frank et al. (2002), shows that the equatorial density falls much more steeply than the equatorial field strength in the radial distance range from $\sim 10$ to $\sim 20 R_{J}$. The theory presented here shows that such profiles are stable only because of the effect of the coupling of the equatorial plasma disk with the ionosphere, through the plasma that lies between. The observed profiles in this region suggest that the ratio of the plasma density inside and outside the plasma disk is $\sim 0.005$. Beyond $\sim 20 R_{J}$, however, the equatorial density is found to fall less steeply with distance than the equatorial field strength, suggesting the action of additional smoothing processes, together possibly with the effect of thinning of the plasma disk.

We finally note that although most of this paper has been concerned specifically with the Jovian system, similar considerations can also be applied in principle to Saturn's magnetospheric plasma environment. This problem will be examined once the field and plasma environment has been sufficiently elucidated through examination of data from the Cassini orbiter mission.

Acknowledgements. This work was partly funded by INTAS grant number 03-51-3922. Work at Nizhny Novgorod was also supported by RFBR grant 05-02-16350, and by the General Physics Division of the Russian Academy of Sciences (Programme "Plasma Processes in the Solar System"). Work at Leicester was also supported by PPARC grant PPA/G/O/2003/00013. Galileo magnetic field data employed in the study were obtained through the Planetary Data System at UCLA.

Topical Editor I. A. Daglis thanks J. Woch and another referee for their help in evaluating this paper.

\section{References}

Acuña, M. H., Behannon, K. W., and Connerney, J. E. P.: Jupiter's magnetic field and magnetosphere, in: Physics of the Jovian Magnetosphere, edited by: Dessler, A. J., Cambridge Univ. Press, Cambridge, U.K., 1-50, 1983.

Bagenal, F.: Empirical model of the Io plasma torus - Voyager measurements, J. Geophys. Res., 99, 11 043-11 062, 1994.

Bagenal, F.: The ionization source near Io from Galileo wake data, Geophys. Res. Lett., 24, 2111-2114, 1997.

Bagenal, F. and Sullivan, J. D.: Direct plasma measurements in the Io torus and inner magnetosphere of Jupiter, J. Geophys. Res., 86, 8447-8466, 1981.

Belcher, J. W.: The low-energy plasma in the jovian magnetosphere, in Physics of the Jovian Magnetosphere, edited by: Dessler, A. J., Cambridge Univ. Press, Cambridge, U.K., 68-105, 1983.

Bespalov, P. A. and Zheleznyakov, V. V.: Formation of disks around hot stars under the action of radiation pressure, Sov. Astron. Lett., 16, 442-452, 1990.

Bespalov, P. A. and Davydenko, S. S.: On the structure of the plasma disk in the Jovian magnetosphere, Planet. Space Sci., 42, 583-592, 1994.

Brown, M. E.: Observations of mass loading in the Io torus, Geophys. Res. Lett., 21, 10, 847-850, 1994.

Bunce, E. J. and Cowley, S. W. H.: Local time asymmetry of the equatorial current sheet in Jupiter's magnetosphere, Planet. Space Sci., 49, 261-274, 2001.

Caudal, G.: A self-consistent model of Jupiter's magnetodisc including the effects of centrifugal force and pressure, J. Geophys. Res., 91, 4201-4221, 1986.

Connerney, J. E. P., Acuña, M. H., and Ness, N. F.: Modeling the Jovian current sheet and inner magnetosphere, J. Geophys. Res., 86, 8370-8384, 1981. 
Cowley, S. W. H. and Bunce, E. J.: Origin of the main auroral oval in Jupiter's coupled magnetosphere-ionosphere system, Planet. Space Sci., 49, 1067-1088, 2001.

Delamere, P. A. and Bagenal, F.: Modeling variability of plasma conditions in the Io torus, J. Geophys. Res., 108(A7), 1276, doi:10.1029/2002JA009706, 2003.

Divine, N. and Garrett, H. B.: Charged particle distributions in Jupiter's magnetosphere, J. Geophys. Res., 88, 6889-6903, 1983.

Ferrière, K. M. and André, N.: A mixed magnetohydrodynamickinetic theory of low-frequency waves and instabilities in stratified, gyrotropic, two-component plasmas, J. Geophys. Res., 108(A7), 1308, doi:10.1029/2003JA009883, 2003.

Frank, L. A. and Paterson, W. R.: Survey of thermal ions in the Io plasma torus with the Galileo spacecraft, J. Geophys. Res., 106, 6131-6149, 2001.

Frank, L. A. and Paterson, W. R.: Plasmas observed near local noon in Jupiter's magnetosphere with the Galileo spacecraft, J. Geophys. Res., 109, A11217, doi:10.1029/2002JA009795, 2004.

Frank, L. A., Paterson, W. R., and Khuarana, K. K.: Observations of thermal plasmas in Jupiter's magnetotail, J. Geophys. Res., 107, A11003, doi:10.1029/2001JA000077, 2002.

Goertz, C. K.: Io's interaction with the plasma torus, J. Geophys. Res., 85, 2949-2956, 1980.

Gold, T.: Motions in the magnetosphere of the Earth, J. Geophys. Res., 64, 1219-1224, 1959.

Gurnett, D. A., Scarf, F. L., Kurth, W. S., Shaw, R. R., and Poynter, R. L.: Determination of Jupiter's electron density profile from plasma wave observations, J. Geophys. Res., 86, 81998212, 1981.

Hill, T. W.: Interchange stability of a rapidly rotating magnetosphere, Planet. Space Sci., 24, 1151-1154, 1976.

Hill, T. W.: Inertial limit on corotation, J. Geophys. Res., 84, 65546558, 1979.

Hill, T. W.: The jovian auroral oval, J. Geophys. Res., 106, 81018107, 2001.

Hill, T. W., Dessler, A. J., and Maher, L. J.: Corotating magnetospheric convection, J. Geophys. Res., 86, 9020-9028, 1981.

Hill, T. W., Dessler, A. J., and Goertz, C. K.: Magnetospheric models, in Physics of the Jovian Magnetosphere, edited by: Dessler, A. J., Cambridge Univ. Press, Cambridge, U.K., 353394, 1983.

Huang, T. S., and Hill, T. W.: Drift-wave instability in the Io plasma torus, J. Geophys. Res., 96, 14 075-14 083, 1991.

Khurana, K. K.: Influence of solar wind on Jupiter's magnetosphere deduced from currents in the equatorial plane, J. Geophys. Res., 106, 25999-26016, 2001.

Khurana, K. K. and Kivelson, M. G.: Inference of the angular velocity of plasma in the jovian magnetosphere from the sweepback of magnetic field, J. Geophys. Res., 98, 67-79, 1993.

Kivelson, M. G., Khurana, K. K., Russell, C. T., and Walker, R. J.: Intermittent short-duration magnetic field anomalies in the Io torus: evidence for plasma interchange?, Geophys. Res. Lett., 24, 2127-2130, 1997.

Krimigis, S. M. and Roelof, E. C.: Low-energy particle population, in: Physics of the Jovian Magnetosphere, edited by: Dessler, A. J., Cambridge Univ. Press, Cambridge, U.K., 106-156, 1983.

Kronberg, E. A., Woch, J., Krupp, N., Lagg, A., Khurana, K. K., and Glassmeier, K.-H.: Mass release at Jupiter: Substorm-like processes in the jovian magnetotail, J. Geophys. Res., 110, A03211,
doi:10.1029/2004JA010777, 2005.

Liu, W. W.: Centrifugally driven instability of a rotationally dominated magnetodisc, J. Geophys. Res., 103, 4707-4714, 1998.

Mauk, B. H. and Krimigis, S. M.: Radial force balance within Jupiter's dayside magnetosphere, J. Geophys. Res., 92, 99319941, 1987.

Mauk, B. H., Krimigis, S. M., and Lepping, R. P.: Particle and field stress balance within a planetary magnetosphere, J. Geophys. Res., 90, 8253-8264, 1985.

Mauk, B. H., Gary, S. A., Kane, M., Keath, E. P., Krimigis, S. M., and Armstrong, T. P.: Hot plasma parameters of Jupiter's inner magnetosphere, J. Geophys. Res., 101, 7685-7695, 1996.

Melrose, D. B.: Rotational effects on the distribution of thermal plasma in the magnetosphere of Jupiter, Planet. Space Sci., 15, 381-393, 1967.

Mikhailovskii, A. B.: Theory of Plasma Instabilities, Vol. 1. Instabilities of an Inhomogeneous Plasma, Consultants Bureau, New York, 1974.

Nichols, J. D. and Cowley, S. W. H.: Magnetosphere-ionosphere coupling currents in Jupiter's middle magnetosphere: Effect of precipitation-induced enhancement of the ionospheric Pedersen conductivity, Ann. Geophys., 22, 1799-1827, 2004.

Pontius Jr., D. H.: Radial mass transport and rotational dynamics, J. Geophys. Res., 102, 7137-7150, 1997.

Pontius, Jr. D.H. and Hill, T.W.: Departure from corotation of the Io plasma torus: Local plasma production, Geophys. Res. Lett., 9, 1321-1324, 1982.

Pontius Jr., D.H., and Hill, T.W.: Rotation-driven plasma transport: The coupling of macroscopic and microdiffusion, J. Geophys. Res., 94, 15 041-15 053, 1989.

Pontius Jr., D.H., Hill, T. W., and Rassbach, M. E.: Steady-state plasma transport in a corotation-dominated magnetosphere, Geophys. Res. Lett., 13, 1097-1110, 1986.

Pontius Jr., D.H., Wolf, R. A., Hill, T. W., Spiro, R. W., Yang, Y. S., and Smyth, W. H.: Velocity shear impoundment of the Io plasma torus, J. Geophys. Res., 103, 19935-19946, 1998.

Sands, M. R. and McNutt, R. L.: Plasma bulk flow in Jupiter's dayside middle magnetosphere, J. Geophys. Res., 93, 8502-8518, 1988.

Siscoe, G. L. and Summers, D.: Centrifugally-driven diffusion of iogenic plasma, J. Geophys. Res., 86, 8471-8479, 1981.

Siscoe, G. L., Eviatar, A., Thorne, R. M., Richardson, J. D., Bagenal, F., and Sullivan, J. D.: Ring current impoundment of the Io plasma torus, J. Geophys. Res., 86, 8480-8484, 1981.

Southwood, D. J. and Kivelson, M. G.: Magnetospheric interchange instability, J. Geophys. Res., 92, 109-116, 1987.

Southwood, D. J. and Kivelson, M. G.: Magnetospheric interchange motion, J. Geophys. Res., 94, 299-308, 1989.

Staines, K., Balogh, A., Cowley, S. W. H., Edwards, T. M., Forsyth, R. J., and Hynds, R. J.: An overview of the Anisotropy Telescope observations of $\mathrm{MeV}$ ions during the Ulysses Jupiter encounter, Planet. Space Sci., 44, 341-369, 1996.

Summers, D. and Siscoe, G. L.: Wave modes of the Io plasma torus, Astrophys. J., 295, 678-684, 1985.

Thorne, R. M., Armstrong, T. P., Stone, S., Williams, D. J., McEntire, R. W., Bolton, S. J., Gurnett, D. A., and Kivelson, M. G.: Galileo evidence for rapid interchange transport in the Io torus, Geophys. Res. Lett., 24, 2131-2134, 1997.

Vasyliunas, V. M.: Plasma distribution and flow, in Physics of the 
Jovian Magnetosphere, edited buy: Dessler, A. J., Cambridge Univ. Press, Cambridge, U.K., 395-453, 1983.

Woch, J., Krupp, N., and Lagg, A.: Particle bursts in the jovian magnetosphere: Evidence for a near-Jupiter neutral line, Geophys. Res. Lett., 29(7), doi:10.1029/2001GL014080, 2002.
Woch, J., Krupp, N., Lagg, A., and Tomás, A.: The structure and dynamics of the Jovian energetic particle distribution, Adv. Space Res., 33, 2030-2038, 2004.

Yang, Y. S., Wolf, R. A., Spiro, R. W., Hill, T. W., and Dessler, A. J.: Numerical simulation of torus-driven transport in the Jovian magnetosphere, J. Geophys. Res., 99, 8755-8770, 1994. 